\title{
IDENTIDAD Y EQUIDAD DE GÉNERO EN LA EDUCACIÓN DE LA PRIMERA INFANCIA
}

\author{
GENDER IDENTITY AND GENDER EQUITY \\ IN EARLY CHILDHOOD EDUCATION
}

\author{
Patricia I. Estrella Romero \\ Educadora de Párvulos PUCV \\ Magister en Evaluación Educacional UPLA \\ Directora Nacional OMEP Chile \\ pestrellar@gmail.com
}

Resumen: El siguiente formato de reflexión recurre a los actores claves de la primera infancia, puesto que surge de un análisis colectivo en OMEP Chile, en base a la siguiente interrogante ¿cómo nos interpela el movimiento feminista nacional en las demandas de equidad de género, a quienes trabajamos diariamente por lograr una educación de calidad en la primera infancia? Sin duda que existe un compromiso y una gran responsabilidad de los profesionales de la educación frente a la temática de género, para avanzar en una perspectiva integradora y de reconocimiento de las múltiples acciones que debemos emprender. Por tanto, esta reflexión presenta los avances y los desafíos frente a la identidad y equidad de género en la educación de la primera infancia en Chile, con algunos datos relevantes actualizados, para impulsar diversas de instancias de análisis, discusión y debate de lo realizado, en pos del logro de los objetivos de la Educación para el Desarrollo Sostenible (EDS).

Palabras clave: educación primera infancia, género, identidad de género, equidad de género, estereotipos de género, EDS.

Abstract: The present reflection takes into consideration key actors of early childhood, since it emerges from a collective analysis in OMEP Chile, based on the following question: how does the national feminist movement challenge in terms of gender equity to those of us who work daily to achieve quality in early childhood education? Undoubtedly, there is a commitment and a great responsibility among the professionals of education regarding the issue of gender in order to advance in an integrating perspective and recognition of the multiple actions that we must undertake. Therefore, this article presents the advances, as well as the challenges, in addressing gender identity and equity in early childhood education in Chile, based on relevant updated data, to promote diverse instances of analysis, discussion and debate of what has been done, in pursuit of the achievement of the objectives of Education for Sustainable Development (ESD).

Key words: early childhood education, gender, gender identity, gender equity, gender stereotypes, EDS. 


\section{INTRODUCCIÓN}

En el transcurso del presente año en Chile, se han presenciado diversas manifestaciones del movimiento feminista como acciones demandantes a la sociedad y sus autoridades. Las manifestantes han expresado con fuerza que se requieren transformaciones, aduciendo "una educación no sexista, equidad de género y fin a la violencia machista ${ }^{1}$...en la cual "están involucradas las mujeres indígenas, subcontratadas, inmigrantes, las trabajadoras...No vamos a paralizar esto, hasta que logremos un cambio cultural".

Los cambios culturales son procesos que provocan desafíos, encuentros y desencuentros, manifestados en el interés de profundizar en temáticas de identidad y equidad de género desde variados ámbitos, instando a que cada persona asuma su cuota de responsabilidad y de compromiso frente a esta causa generada por el movimiento feminista, y que representa concretamente una vulneración de derechos humanos.

Desde la perspectiva del nivel etario que abarca la Educación de la Primera Infancia, esta demanda se suma y se relaciona directamente con el debate nacional situado entre las problemáticas que afectan a la niñez en la actualidad, especialmente a la niñez vulnerada en sus derechos. Y uno de ellos, el derecho a la identidad, en específico "identidad y equidad de género", es el foco de la presente reflexión.

En Educación Parvularia, el derecho a la identidad ha formado parte esencial desde sus orígenes. La Ley General de Educación Chilena ${ }^{2}$ establece explícitamente que "es el nivel educativo que atiende integralmente a niños desde su nacimiento hasta su ingreso a la educación básica...".

\section{PRIMERA INFANCIA Y GÉNERO}

\section{Referencias globales y locales}

Algunos antecedentes históricos que han avanzado en esta línea, son los expresados en la Convención de los Derechos del Niño $\left(C D N^{3}\right)$, en que los Estados asumen el compromiso de "respetar el derecho del niño a preservar su identidad, incluidos la nacionalidad, el nombre y las relaciones familiares de conformidad con la ley sin injerencias ilícitas" (Art. 8.1). Y, los formulados por el Comité de Derechos del Niño, que ha agregado recientemente "características como el sexo, la orientación sexual, el origen nacional, la religión y las creencias, la identidad cultural y la personalidad".

Sumado a ello, la Convención (CDN, Artículo 2) señala diversos motivos respecto a los cuales está prohibido discriminar, en particular "la raza, el color, el sexo, el idioma, la religión, la opinión política o de otra índole, el origen nacional, étnico o social, la posición económica, los impedimentos físicos, el nacimiento o cualquier otra condición del niño, de 
sus padres o de sus representantes legales". Así como también la orientación sexual, la identidad de género y el estado de salud, en particular el VIH/SIDA y la salud mental ${ }^{5}$.

Por su parte, la Declaración de Goteborg6, en específico en sus recomendaciones generales relativas a Género (GENDER), establece que "la Educación para el Desarrollo Sostenible (EDS) debe promover activamente el compromiso crítico con las normas que definen las formas de género de ser, hacer y convivir, y debe valorar particularmente el papel y la contribución de las mujeres para lograr un cambio social y garantizar el bienestar humano".

La Agenda 2030 para el Desarrollo Sostenible ${ }^{7}$, que establece 17 Objetivos y 169 metas a ser implementados tanto a nivel nacional, regional y global, presenta una visión del desarrollo sostenible e integra sus dimensiones económica, social y ambiental, para abordar los desafíos estructurales y las prioridades de América Latina y el Caribe.

Esta Agenda y sus Objetivos representan una gran oportunidad para lograr la igualdad de género y la autonomía de las mujeres. Para ello, la Agenda incluye el Objetivo 5, denominado "Lograr la igualdad de género y empoderar a todas las mujeres y las niñas". De igual forma, la Agenda incorpora transversalmente la perspectiva de género en las metas de los demás Objetivos de Desarrollo Sostenible.

Estos objetivos y metas deben ser alineados y complementados con los compromisos ya existentes en la Agenda Regional de Género, que son el denominador común regional.

En relación a ello, la "Agenda de Equidad de Género del Gobierno de Chile", en el Ministerio de la Mujer y la Equidad de Género ${ }^{8}$, considera una serie de 23 medidas que esperan lograr acuerdos y avances en materia de igualdad de derechos de mujeres y hombres en nuestra sociedad. $Y$ una de esas medidas urgentes, por ejemplo, es el Derecho a Sala Cuna para todos los niños y niñas de madres o padres trabajadores al cuidado de sus hijos.

Actualmente las políticas de gobierno contemplan la priorización de la niñez vulnerada en sus derechos estableciendo un "Acuerdo Nacional por la Infancia" ${ }^{\text {, }}$ con una serie de medidas que permitan prevenir vulneraciones a través de un sistema de alerta temprana y educación preventiva (abuso sexual infantil, drogas y alcohol) y, promover el máximo desarrollo de capacidades de los niños a través de acciones oportunas, como por ejemplo, Educación Parvularia universal y de calidad"10.

Por su parte, desde la Comisión Mixta de la Cámara del Senado Chileno, se dio inicio en el presente año a la discusión del proyecto sobre derecho a la educación y equidad de género -definiendo con votación de mayoría - la identidad de género como un derecho ${ }^{11}$. 


\section{Integración de las directrices de OMEP con las Bases Curriculares chilenas}

A partir del quehacer propio, OMEP (Organización Mundial para la Educación Preescolar) se ha sumado a los Objetivos de Desarrollo Sostenible (ODS) ${ }^{12}$ adoptados por los líderes mundiales en el año 2015, enfatizando la necesidad de lograr la igualdad entre los géneros y empoderar a todas las mujeres y niñas del mundo.

Tales objetivos se adoptan y formulan en las Declaraciones Mundiales de OMEP, atendiendo a las problemáticas que impactan la vida de las niñas y los niños en la primera infancia, promoviendo la reflexión y acción de Educadores, familias y comunidades educativas, autoridades gubernamentales, entre otros actores relevantes en este ámbito, a fin de avanzar en el cumplimiento de los objetivos propuestos.

A continuación, se especifican objetivos y metas planteadas en las Declaraciones Mundiales de OMEP en este decenio, a saber:

- "Empoderar a las niñas y mujeres y lograr igualdad de género", y la Meta recomendada: "Comenzar el empoderamiento y la igualdad de género en la primera infancia, centrándose en el acceso de las niñas a una educación preescolar de calidad, segura, no violenta y no discriminatoria ${ }^{13}$. (OMEP, 2013)

- "Asegurar igualdad de derechos a la educación y a los cuidados para todos los niños y niñas, dando prioridad a los más pobres y más vulnerables, incluyendo a los que viven en áreas de conflicto y como refugiados"14. (OMEP, 2014)

- "Garantizar que todas las niñas y los niños tengan acceso a un desarrollo de calidad en la primera infancia, al cuidado y a la educación infantil..." La meta es cerrar las brechas que existen en la actualidad para que todos los niños puedan alcanzar su pleno potencial"15. (OMEP, 2015)

- "Garantizar condiciones de vida justa y humanitaria para todos los niños desplazados, migrantes, refugiados o solicitantes de asilo"16. (OMEP, 2016)

- "Asignar los recursos necesarios para garantizar la equidad y la calidad en la $\mathrm{AEPI}^{17}$, ampliando los derechos de los sectores más desfavorecidos" ${ }^{18}$. (OMEP, 2017)

- "Iniciar más proyectos con niños, especialmente relacionados con la Educación para el Desarrollo Sostenible..." (Asamblea Mundial de OMEP, Praga, 2018)

Para el logro de tales objetivos, OMEP demanda a los gobiernos dar cumplimiento a los compromisos financieros relacionados con el desarrollo y la sustentabilidad de la educación de la primera infancia. 
Como parte de ese llamado se pueden apreciar ciertos avances, en particular, en el ámbito de la primera infancia en Chile es posible apreciar la temática "identidad de género" en las nuevas Bases Curriculares de la Educación Parvularia (BCEP) ${ }^{19}$.

En este referente curricular, de los ocho propósitos expuestos, en el tercero y quinto respectivamente se declara: "Promover en la niña y el niño la identificación y valoración progresiva de sus propias características personales, necesidades, preferencias y fortalezas, para favorecer una imagen positiva de sí mismos y el desarrollo de su identidad y autonomía, así como la consideración y respeto hacia los demás" y, "Propiciar aprendizajes de calidad en las niñas y los niños que sean pertinentes y consideren las necesidades educativas especiales, las diversidades culturales, lingüísticas, de género, religiosas y sociales, junto a otros aspectos culturales significativos de ellos, sus familias y comunidades". (BCEP, pp.33-34)

Seguidamente, en el contexto de las interacciones que establecen los niños y niñas con otras personas significativas, "los párvulos comienzan a tomar conciencia gradual de sus características y atributos personales, entre ellos su sexo e identidad de género, sus fortalezas, habilidades, apegos, intereses y preferencias. A la vez que afirman su identidad, requieren más autonomía". (BCEP, p.47)

En las nuevas Bases Curriculares (BCEP) se considera la educación inclusiva, "con especial énfasis en aquellos quienes requieren mayor protección, tales como: pueblos indígenas, migrantes, poblaciones rurales, diversidad sexual y de género, privados de libertad, con discapacidad, con alguna enfermedad y con dificultades de aprendizaje, para brindar oportunidades de aprendizaje a todos los párvulos, jóvenes y adultos". (p.22)

A su vez, desprendidos de la Ley General de Educación ${ }^{20}$, se contemplan aspectos propios de la identidad de género en 2 de los 13 objetivos generales, a saber: "Apreciar sus capacidades y características personales" y, "Desarrollar actitudes de respeto y aceptación de la diversidad social, étnica, cultural, religiosa y física" (Artículo 28, letras b y e, p.16 en BCEP).

\section{Algunas experiencias en Chile}

En el marco de la educación inclusiva, es notable considerar experiencias acumuladas por las diversas instituciones relacionadas con la primera infancia en Chile, ya que tanto JUNJI ${ }^{21}$ como INTEGRA ${ }^{22}$ (a través de sus sitios web) revelan y comparten el trabajo que vienen realizando desde hace años, integrando el enfoque o perspectiva de género en sus referentes curriculares, sensibilizando a las comunidades educativas y avanzando hacia una sociedad más equitativa.

Tales menciones, se suman a una serie de iniciativas del Ministerio de Educación en Chile - como la Unidad de Equidad de Género (UEG) ${ }^{23}$ - que relevan la necesidad de acoger esta temática en todos los niveles educativos, habiendo formulado para el periodo 
2015-2018 el Plan “Educación para la Igualdad entre Hombres y Mujeres", que tiene como objetivos "promover la igualdad y el desarrollo integral de hombres y mujeres en el sistema educacional; establecer mecanismos y competencias del Ministerio de Educación; y promover propuestas para la inclusión de la perspectiva de género en la Reforma Educacional"24.

En el mencionado Plan ${ }^{25}$, se establece una Hoja de Ruta para la incorporación del enfoque de género en educación, con las siguientes líneas de acción: Fortalecimiento de competencias en funcionarios/as y actores del sistema educativo; Difusión, sensibilización e información; Producción de conocimientos y herramientas; Articulación de actores.

Y seguidamente, como medidas de equidad diseñadas para el nivel de Educación Parvularia, se indican las siguientes:

- "Resguardo de lenguaje inclusivo, detección de estereotipos, desarrollo de la identidad, entre otros, en la actualización de las bases curriculares, programas pedagógicos, materiales pedagógicos y marcos orientadores para los equipos educativos.

- Fortalecimiento de capacidades de los equipos que diseñan las políticas actuales y equipos técnicos de las instituciones prestadoras de servicio (Junji e Integra)"26.

Por su parte, la Agencia de Calidad de la Educación expresa... "Invitamos a todas las escuelas y las familias del país a no encasillar a niños y niñas en habilidades y oportunidades desprendidas de estereotipos, los que restringen el potencial de los estudiantes; aún más, limitan las posibilidades de desarrollo de un país entero"27.

Y uno de los elementos reflexivos que emergen en esta invitación son los estereotipos, frente a los cuales hay un gran desafío sociocultural, en especial para quienes trabajan en la educación de la primera infancia. Desde la Subsecretaría de Educación Parvularia se ha publicado un texto relativo a la Igualdad de Género ${ }^{28}$, en el cual se presentan normativas, conceptos y acciones que orientan a los equipos pedagógicos de los establecimientos educacionales, contribuyendo a generar condiciones para abordar estos tópicos transversales en la formación de los niños y niñas de esta etapa educativa.

Respecto a los estereotipos de género, se señala en el citado documento, que "están en la cotidianeidad de los procesos educativos que redundan en las brechas de resultados y se encuentran presentes en el currículum y en los cargos de las y los docentes; en la participación y liderazgo del estudiantado en diversas actividades; en las actitudes de los y las estudiantes y en las expectativas e influencias familiares; en la formación inicial de las y los futuros docentes y en sus posteriores prácticas pedagógicas, entre otros; es así que estas desigualdades de género permean la educación y se 
encuentran en los resultados de las pruebas estandarizadas y en las futuras elecciones de jóvenes, mujeres y hombres, al definir sus proyectos de vida" 29 .

Una muestra de ello, se encuentra en los variados indicadores de género expuestos por el Instituto Nacional de Estadísticas en Chile (INE), los cuales revelan que "Durante el periodo 2007-2016, las mujeres representaron -a nivel nacional- menos del $\mathbf{2 5 \%}$ del total de personas tituladas en carreras profesionales del área de tecnología. En 2016 fueron $\mathbf{2 4 , 6 \%}$, con una brecha de $-50,8$ puntos porcentuales ${ }^{30}$ (ver Gráfico 1).

\section{Gráfico 1.}

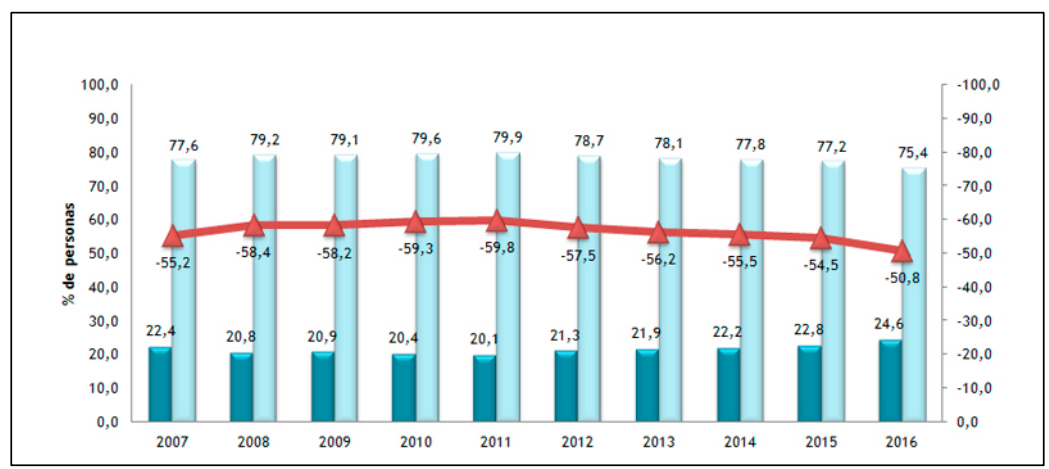

\section{Algunos conceptos relevantes para comenzar el diálogo}

Para situar la necesaria discusión y reflexión de este tema es relevante considerar conceptos centrales de esta temática de modo de establecer un lenguaje común, para llegar a profundizar en la formación de las Educadoras de Párvulos y Técnicos de Educación Parvularia, puesto que es sabido que en la interacción diaria con los párvulos, con sus familias, con los equipos pedagógicos y la comunidad educativa, se transparentan y afloran los estereotipos asociados a la construcción de la identidad de género en la primera infancia.

Las acciones intencionadas pedagógicamente a emprender por los equipos profesionales y técnicos, permitirán avanzar en la equidad de género y en la disminución de las brechas entre hombres y mujeres en el presente cotidiano y en el futuro de sus vidas como ciudadanos. Por tanto, existe desde ya para la educación de la primera infancia, una gran responsabilidad, enormes desafíos y compromiso en la construcción de la identidad e igualdad de género.

Para avanzar en un proceso dialógico y reflexivo necesario en los equipos pedagógicos, a continuación se hace referencia a ocho conceptos esenciales de esta temática - de otros tantos presentes - a considerar:

Género, entendido como "el conjunto de características sociales y culturales en torno a los femenino/masculino. Está conformado por ideas, creencias y atribuciones 
culturales e históricamente situadas en relación con el sexo biológico. El concepto de género es prescriptivo, es decir, constituye un sistema de roles que define un modo único y excluyente de ser mujer y de ser hombre" (Mineduc, s.f., p.17)

Sexo, se refiere a "la clasificación de las personas al momento de nacer, como hombre, mujer o intersex, en función de sus características biológicas y anatómicas" (Mineduc, 2017)

Identidad de género, definido como "la concepción individual de género que tiene una persona de sí misma y que no tiene por qué depender necesariamente del género que le fue asignado al nacer" (Mineduc, s.f.; p.19). A los cuatro años de edad, la mayoría tiene un sentido establecido sobre su identidad de género ${ }^{31}$.

Igualdad de género, relativo a "la igualdad de derechos y oportunidades para todas las personas, sin distinciones en función del género. La igualdad no significa considerar a mujeres y hombres iguales en características, sino en derechos. Equivale a decir también que sobre las diferencias sexuales no se pueden cimentar desigualdades sociales" (Mineduc, s.f., Mineduc, 2017).

Equidad de género, se define como "un medio para alcanzar la igualdad de género. Refiere al trato justo para hombres, mujeres o cualquier variante de género en función de sus respectivas necesidades y considerando los contextos culturales" (Mineduc, s.f.) ${ }^{32}$

Brecha de género, entendida como "un indicador cuantitativo o cualitativo de las desigualdades entre hombres y mujeres en ámbitos como la participación, el acceso a oportunidades, el rendimiento académico, uso del espacio, etc. Cuanto menor es la brecha de género, más cercano se está hacia la igualdad".

Estereotipos de género, "son creencias y atributos generalizados que se hacen sobre un grupo de personas en función de su género y en relación a asuntos como la emocionalidad, la capacidad física e intelectual, la sexualidad, los intereses, etc. De los estereotipos de género emergen prejuicios hacia ciertos grupos de personas y valorizaciones diferenciadas que derivan en prácticas de discriminación" (Mineduc, s.f.)

Pedagogía invisible de género (o currículo oculto), "comprende un conjunto de normas, valores y creencias relativas a las desigualdades de género, no afirmadas de manera explícita, que son transmitidas a los y las estudiantes por medio del contenido formal y de las relaciones que se establecen durante la vida escolar"33.

\section{Ciertas propuestas de encuentro.}

Para abordar la temática de identidad e igualdad de género, la Subsecretaría de Educación Parvularia, ha generado una propuesta metodológica de trabajo necesaria de 
reflexionar con los equipos pedagógicos y con las familias de los niños y niñas, distinguiendo los siguientes pasos:

a) Generar conciencia sobre esta materia: conociendo, problematizando y reflexionando acerca de los principales conceptos y qué significa en la práctica construir una Educación Parvularia que promueva la igualdad;

b) Sensibilizar y suscitar un trabajo intencionado con los equipos, creando instancias que permitan revisar las propias experiencias de vida, crianza y estilo de educación recibido, a fin de ir reconociendo los prejuicios y estereotipos de género;

c) Observar y analizar la práctica cotidiana, realizando tanto un trabajo personal como grupal, que junto a las acciones anteriores, se suman a las primeras aproximaciones para desarrollar una pedagogía con enfoque de género $y$ encaminada hacia la igualdad de género.

A esta propuesta se agrega lo planteado por Camacho y Watson ${ }^{34}$, determinando que la "equidad de género debe ser un eje transversal, porque no es una materia que se enseña, sino más bien una forma de mirar el mundo y de vivir..."

Para ello, sugieren los autores señalados, considerar una serie de ejes, temáticas o ideas organizadoras que sirven como base para el desarrollo de contenidos de género en un aula y que permitirán construir relaciones equitativas de género desde el nivel inicial, tales como:

- $\quad$ Igualdad de oportunidades.

- Igualdad de responsabilidades.

- Igualdad en la valoración de las diferencias.

- $\quad$ El trabajo.

- Los roles domésticos y familiares.

- Los vínculos sociales, afectivos y amorosos.

- $\quad$ El compromiso ante la maternidad/ paternidad

- $\quad$ La realización personal y proyectos de vida.

Así, es necesario que la temática de género transite por todas las acciones e interacciones del proceso educativo, generando un currículo que responda a las necesidades e intereses de los niños y niñas, que busque y ofrezca igualdad de condiciones y oportunidades para todos y todas.

Por su parte, con el propósito de visibilizar brechas, barreras e inequidades de género en ámbitos imperiosos para el logro de la autonomía personal y la igualdad de género, el Instituto Nacional de Estadísticas (INE), ha generado algunos indicadores prioritarios de seguimiento. Por su relevancia en la formación de las/los profesionales de la educación de la primera infancia, y por el sentido de urgencia en el análisis y debate relativo a la "identidad de género", a continuación se especifican aquellos indicadores: 
a) Autonomía física: relacionada con la capacidad de control sobre el propio cuerpo (Indicadores: violencia contra las mujeres, maternidad adolescente);

b) Autonomía en la toma de decisiones: plena participación en las decisiones que afectan a su vida y a su colectividad (Indicadores de participación en gobiernos locales y regionales, poder judicial, poder legislativo, organizaciones sociales, poder ejecutivo);

c) Autonomía económica: capacidad de las mujeres para generar ingresos propios y controlar los activos y recursos (mercado del trabajo, emprendimiento, carga global de trabajo, entre otros);

d) Procesos de aprendizaje para el cambio cultural: relacionado con procesos asociados a los ámbitos educativos formales e informales, y de socialización, como los medios de comunicación (participación en carreras universitarias, rendimiento).

Y de este último indicador por ejemplo, emergen datos de la participación de la mujer en carreras universitarias, en específico en el "Atlas de Género" ${ }^{35}$, visibilizando las brechas entre ambos sexos respecto del nivel de ingreso, variables sociodemográficas y ocupacionales en el período 2010 - 2016.

Muy a pesar, y como se señala en INE (2018) "aún persisten amplias brechas de género en el ingreso de las Personas Ocupadas y visibilizan uno de los grandes desafíos a nivel país para cumplir con la Agenda 2030 de Naciones Unidas y su objetivo de lograr la igualdad entre los géneros y empoderar a todas las mujeres y niñas ${ }^{36}$.

\section{REFLEXIONES}

Los resultados expuestos reflejan lo mucho que aún queda por avanzar y, en específico, el rol que le compete a la Educación de la Primera Infancia, puesto que "debe propiciar ambientes de aprendizaje que no reproduzcan estereotipos, ni sostengan sesgos de género y/o prácticas discriminatorias" ${ }^{37}$.

El avance en materia de igualdad de género, requiere de una red colaborativa más intensa e intencionada en este ámbito, generando a su vez diversidad de espacios de reflexión y debate que demuestren a la sociedad los logros alcanzados en los objetivos mundiales, regionales y locales relativos a la "identidad y equidad de género".

En síntesis, se precisa generar espacios de expresión (Seminarios, Congresos, Publicaciones, entre otros), que realcen las acciones ejercidas por los profesionales de la educación de la primera infancia, frente al compromiso y responsabilidad que les incumbe en el "cambio cultural" demandado por las manifestantes del movimiento feminista en Chile. Como ejemplo final, actualmente la Universidad de Chile abre la discusión sobre cómo materializar la educación no sexista ${ }^{38}$.

El espacio reflexivo de este artículo, ha permitido visualizar una aproximación directa frente a la vulneración de derechos, en ambas partes. 


\section{NOTAS}

1) http://www.elmostrador.cl/noticias/pais/2018/06/06/movimiento-feministasigue-rugiendo-logra-nueva-marcha-masiva-por-la-alameda/

2) Ley General de Educación - Ley 20.370 12-septiembre-2009

3) Convención de los Derechos del Niño (ONU 1989) Chile Ley promulgada 1990 https://www.leychile.cl

4) Biblioteca del Congreso Nacional de Chile (BCN) - Documento Asesoría Técnica Parlamentaria (octubre/2017), (CRC, 2013b:párr. 55).

5) Biblioteca del Congreso Nacional de Chile (BCN) - Documento Asesoría Técnica Parlamentaria (octubre/2017), (CRC, 2103a:párr.8).

6) The Gothenburg Recommendations on Education for Sustainable Development. Adopted November 12, 2008

7) http://biblioguias.cepal.org/AgendaRegionalGenero/Agenda2030

8) https://www.minmujeryeg.cl/agenda-mujer/\#

9) https://infancia.sebastianpinera.cl/

10) https://www.cepchile.cl/acuerdo-nacional-por-la-infancia/cep/2018-0604/113232.html

11) Senado de la República - Comisión Mixta, Boletín № 11906-04. Equidad de género. $(11 / 07 / 2018)$

12) https://www.pactoglobal.cl/objetivos-de-desarrollo-sostenible-ods/

13) OMEP 2013 - Resolución: "Importancia de la primera infancia como alta prioridad para la Agenda Post 2015". Objetivo 2

14) Declaración de la 66a. Asamblea y Conferencia Mundial de OMEP, Julio 2014 Cork, Irlanda. En reconocimiento al $25^{\circ}$ aniversario de la Convención sobre los Derechos del Niño de Naciones Unidas.

15) Declaración de la 67ạ Asamblea Mundial y Conferencia de la OMEP "La clave para el futuro del mundo: los educadores de la primera infancia" Julio de 2015, Washington, D.C. Los Objetivos de Desarrollo Sostenible de la ONU para 2015 2030, incluyen tal objetivo.

16) Declaración de la $68^{\circ}$ Asamblea y Conferencia Mundial de la OMEP "Llamamiento urgente a considerar los derechos y necesidades de niños pequeños desplazados, migrantes, refugiados, y/o solicitantes de asilo" Seúl, Corea, julio de 2016.

17) AEPI "Atención y Educación en la Primera Infancia"

18) Declaración de la $69^{\circ}$ Asamblea y Conferencia Mundial de la OMEP "Mayor inversión para la atención y educación de la primera infancia (AEPI) Opatija, Croacia, 20 de junio de 2017.

19) Mineduc, Subsecretaría de Educación Parvularia, BCEP, febrero 2018

20) LGE Ley General de Educación № 20.370

21) JUNJI "Guía para incorporar el enfoque de género en las prácticas educativas en JUNJI https://www.junji.gob.cl/wp-content/uploads/2016/06/Guia-paraincorporar-el-Enfoque-de-Genero-en-las-practicas-educativas-de-JUNJI.pdf 
22) xxii.http://bibliotecas.integra.cl/cedoc/opac_css/index.php?lvl=categ_see\&id=234 \&main=

23) MINEDUC - UEG (Unidad de Equidad de Género creada en Marzo de 2015)

24) MINEDUC - UEG https://www.mineduc.cl/wpcontent/uploads/sites/19/2017/01/CartillaUEG.pdf

25) MINEDUC - “Educación para la Igualdad de Género Plan 2015-2018”, p.26

26) MINEDUC - https://www.mineduc.cl/wpcontent/uploads/sites/19/2017/01/CartillaUEG.pdf p.27

27) Agencia de Calidad de la Educación, Carlos Henríquez C. Secretario Ejecutivo en artículo “Educación y brecha de género" 09.marzo.2018

28) MINEDUC, Subsecretaría de Educación Parvularia, División de Políticas Educativas "Orientaciones para promover la igualdad de género en Educación Parvularia".Marzo,2018

29) MINEDUC, Subsecretaría de Educación Parvularia, División de Políticas Educativas "Orientaciones para promover la igualdad de género en Educación Parvularia".Marzo, 2018. p.10

30) http://www.ine.cl/estadisticas/menu-sociales/genero

31) Fundación Todo Mejora, Manual "Enseñando diversidad"(2017) https://todomejora.org/wpcontent/uploads/2017/03/ensenando_diversidad_TM.pdf.

32) MINEDUC, Subsecretaría de Educación Parvularia, División de Políticas Educativas "Orientaciones para promover la igualdad de género en Educación Parvularia".Marzo,2018, p.19

33) REDALYC, 2008 Artículo "Reflexiones sobre equidad de género y educación inicial". Camacho y Watson (Abarca, 2003b: 12) http://www.redalyc.org/pdf/666/66615071004.pdf

34) REDALYC, 2008 Artículo "Reflexiones sobre equidad de género y educación inicial". Camacho y Watson (Abarca, 2003b: 12) http://www.redalyc.org/pdf/666/66615071004.pdf Inter Sedes. Vol. VIII. (14-2007) 33-48. ISSN: $1409-4746$ p.45

35) http://inechile.maps.arcgis.com/apps/Cascade/index.html?appid=00b769d5f3dc4 $06 \mathrm{ca} 2 \mathrm{~b} 2119 \mathrm{~d} 46018111$

36) http://historico.ine.cl/genero/files/estadisticas/pdf/documentos/genero ingresos 2017.pdf Mayor información sobre la agenda 2030 y sobre este objetivo específicamente consultar la resolución aprobada por Naciones Unidas.http://www.un.org/es/comun/docs/?symbol=A/RES/70/1

37) Id. p.15

38) http://www.elmostrador.cl/braga/2018/07/23/u-de-chile-abre-la-discusion-sobrecomo-materializar-la-educacion-no-sexista/ 


\section{REFERENCIAS BIBLIOGRÁFICAS}

Camacho, L y Watson, H. (2007) Reflexiones sobre equidad de género y educación inicial. InterSedes 8(14): 33-48 http://www.redalyc.org/pdf/666/66615071004.pdf

Henríquez, C. (2018) Educación y brecha de género. Agencia de Calidad de la Educación.

Fundación Todo Mejora (2017) Manual "Enseñando diversidad"

https://todomejora.org/wp-content/uploads/2017/03/ensenando diversidad_TM.pdf

JUNJI (2016) Guía para incorporar el enfoque de género en las prácticas educativas en JUNJI https://www.junji.gob.cl/wp-content/uploads/2016/06/Guia-para-incorporar-elEnfoque-de-Genero-en-las-practicas-educativas-de-JUNJI.pdf

MINEDUC (2018) Bases curriculares de la educación parvularia.

OMEP (2008) The Gothenburg recommendations for sustainable development. 